\title{
Molecular Simulation Research on Domain Micro-structure of TSP-POSS/PU Hybrid Composites
}

\author{
R. Pan*, L.L. Wang \\ Chemistry and Material Science College \\ Sichuan Normal University \\ Chengdu City, PRC, 610041 \\ *Corresponding author
}

\author{
Y. Liu \\ Key Laboratory of Special Waste Water Treatment \\ Sichuan Province Higher Education System \\ Chengdu City, PRC, 610041
}

\begin{abstract}
Trisilanolphenyl polyhedral oligomeric silsesquioxane (TSP-POSS) with three phenyl groups, was incorporated in concentrations of 6, 12 and $20 \mathrm{wt} \%$ into 4, 4'-methylenebis(phenyl isocyanate) (MDI) and glycerol propoxylate to prepare TSP-POSS/PU hybrid composites models, respectively. The domain micro-structures of these hybrid composites models were characterized by mean square displacement and radial distribution function at molecular level. As the result shows: with TSP-POSS concentration increasing, the contacts of polymer chain is decreased and cage cores are inclined to be closer to each other due to the presence of humping cage structure of TSP-POSS unit. Meanwhile, high concentration of TSP-POSS apparently restricts the motion of polymer chains, which also demonstrates that TSP-POSS is as a rigid core linked to the backbones of hybrid composites.
\end{abstract}

Keywords-POSS; polyurethane; molecular dynamics; molecular mechanics

\section{INTRODUCTION}

As nano-particle, polyhedral oligomeric silsesquioxane (abbreviation POSS) has received increasing attention for its inorganic-organic hybrid nature. Most of POSS consists of a totally enclosed cage with eight silica atoms linking together via oxygen atoms.[1] Polymer properties, such as permeability, friction, mechanical and thermal properties, can be significantly altered by introducing different POSS molecules at various concentrations into polymer matrices. [2-5] As with other polymers, POSS can improve the properties of PU in applications ranging from elastomers, coatings to adhesives. However, there is seldom evidence in literature about the influence of TSP-POSS, as one of a few POSS with three phenyl groups, on polyurethane domain micro-structure with covalent incorporation at various concentrations. Since polyurethane has a large range of application and TSP-POSS is a good modification of filler, the mechanism about how the covalent inclusion of TSP-POSS into the PU backbone will tailor the domain micro-structure of hybrid composite is deserved to investigate.

\section{SimUlation}

Accelrys Amorphous Cell module and COMPASS force field in Materials Studio software were adopted in all simulation process in our research, which has been used successfully for the simulation of polymer nanocomposites containing POSS [6-7]. As periodic boundary conditions imposed, an initial low density was used to construct the bulk cubic structures of random hybrid copolymers. 10 initial configurations for each sample were optimized by molecular dynamics technique under the NPT (constant particle numbers, pressure and temperature) conditions at 4Gpa with a minimization involving 30000 steps to relax and equilibrate. After this minimization procedure, the density fluctuation of each system is less than $0.02 \mathrm{~g} / \mathrm{cm}^{3}$ under a given condition. Since these optimized configurations might not be in a local energy minimum state, an annealing procedure from $623 \mathrm{~K}$ to $273 \mathrm{~K}$ was applied on the above optimized configurations by conducting the velocity Verlet algorithm in NVT dynamics to reduce the possible potential energy. Finally, configurations with the highest energy were rejected and 10 configurations of each sample were selected for further analysis of composites' characteristics. The weight fractions of TSP-POSS in composites and sample codes are listed in Table 1. The molecular structures of TSPPOSS /PU hybrid composite are shown in Figure 1. [8]

TABLE I. CHARACTERISTICS OF TSP-POSS/PU HYBRID COMPOSITES.

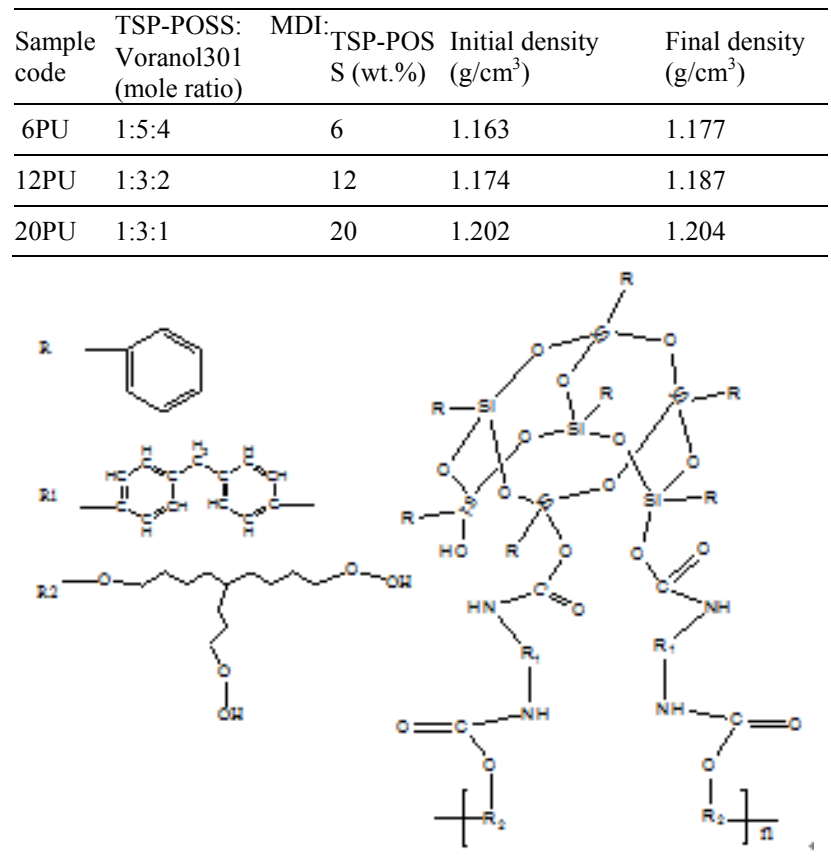

FIGURE I. CHEMICAL STRUCTURES OF TSP-POSS/PU HYBRID COMPOSITES 


\section{RESULTS AND DISCUSSION}

\section{A. Validation of Simulation}

The use of the COMPASS force field for our system was validated by the following method. Recently, it was also reported that the structure and energy of the POSS/PU hybrid composites were successfully simulated by use of the COMPASS force field. Model structure was generated through several cycles of molecular mechanics and molecular dynamics energy minimization. Table 1 lists the characteristic values of all hybrid composites. After the above minimization procedure, the density fluctuation of each system is less than $0.02 \mathrm{~g} / \mathrm{cm}^{3}$ under a given condition, indicating that the structure generated is fully relaxed and is in the equilibrium state which can be confirmed by energy optimization.
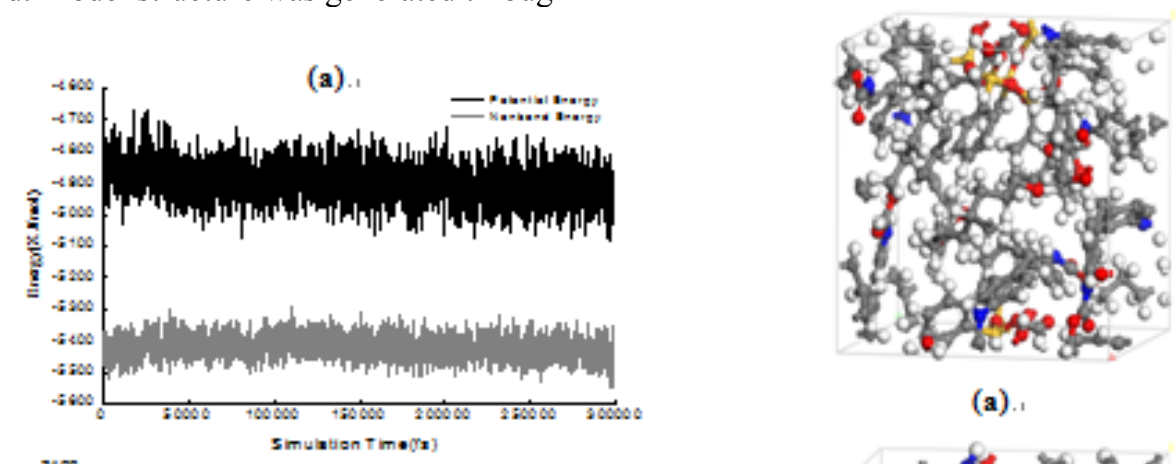

(a). .
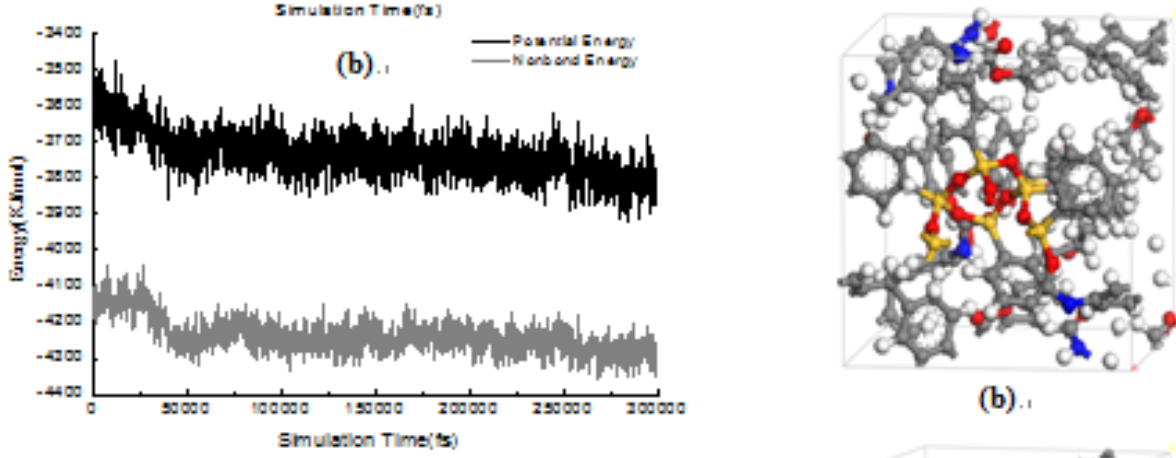

(b).

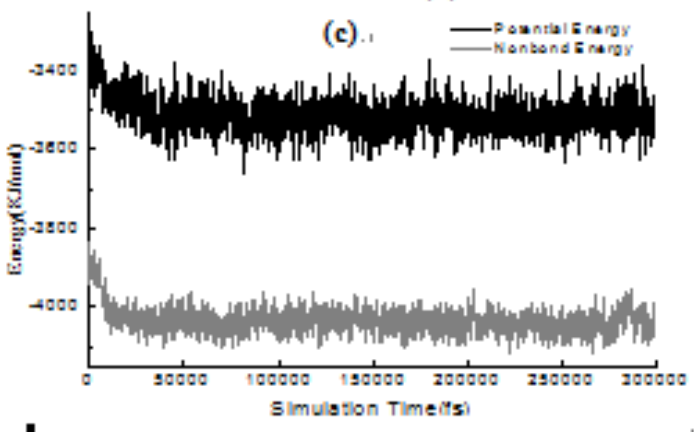

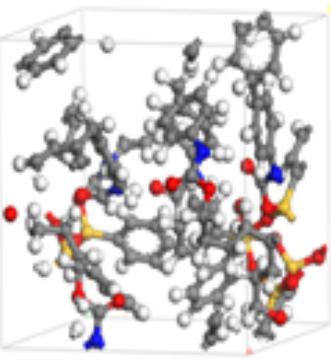

(c).

FIGURE II. ENERGY OPTIMIZATION AND 3D PERIODIC BOUNDARY CONDITIONS OF TSP-POSS/PU HYBRID COMPOSITES: (A). 6PU; (B). 12PU; (C) 20PU.

\section{B. Radial Distribution Function Analysis}

In statistical mechanics, the radial distribution function ( pair correlation function) $\mathrm{g}(\mathrm{r})$ is a measure of the probability of finding a pair of atoms $(\alpha, \beta)$ that is separated by a radial distance $\mathrm{r} \alpha \beta$, which is expected for a completely random distribution. Thus, details of polymer chain packing can be estimated by defining the $\mathrm{Si}-\mathrm{O}$ pair in TSP-POSS core and atoms in polymer main chain as given reference particles in intermolecular pair correlation function, respectively.

Figure3(a) shows the intermolecular pair correlation function based on all backbone atoms in main chains of three samples. No significant diffuse peak can be observed, which indicates no obvious influence on the average spacing between neighboring chains. However, the value of $g(r)$ decreases with increasing concentration of TSP-POSS, which means that at any given distance the number of contacts between neighboring chains is decreased due to the presence of TSP-POSS with humping cage structure [9-10].

Figure 3(b) shows the intermolecular pair correlation function based on atoms $(\mathrm{Si}, \mathrm{O})$ of TSP-POSS cores in composite versus the atoms in the main chain. With the increase of TSP-POSS concentration, the number of contacts between TSP-POSS cores and main chains is slightly increased. It can be concluded that as the concentration increasing, TSP-POSS cores are inclined to be closer due to the inter-cage structure interactions. 

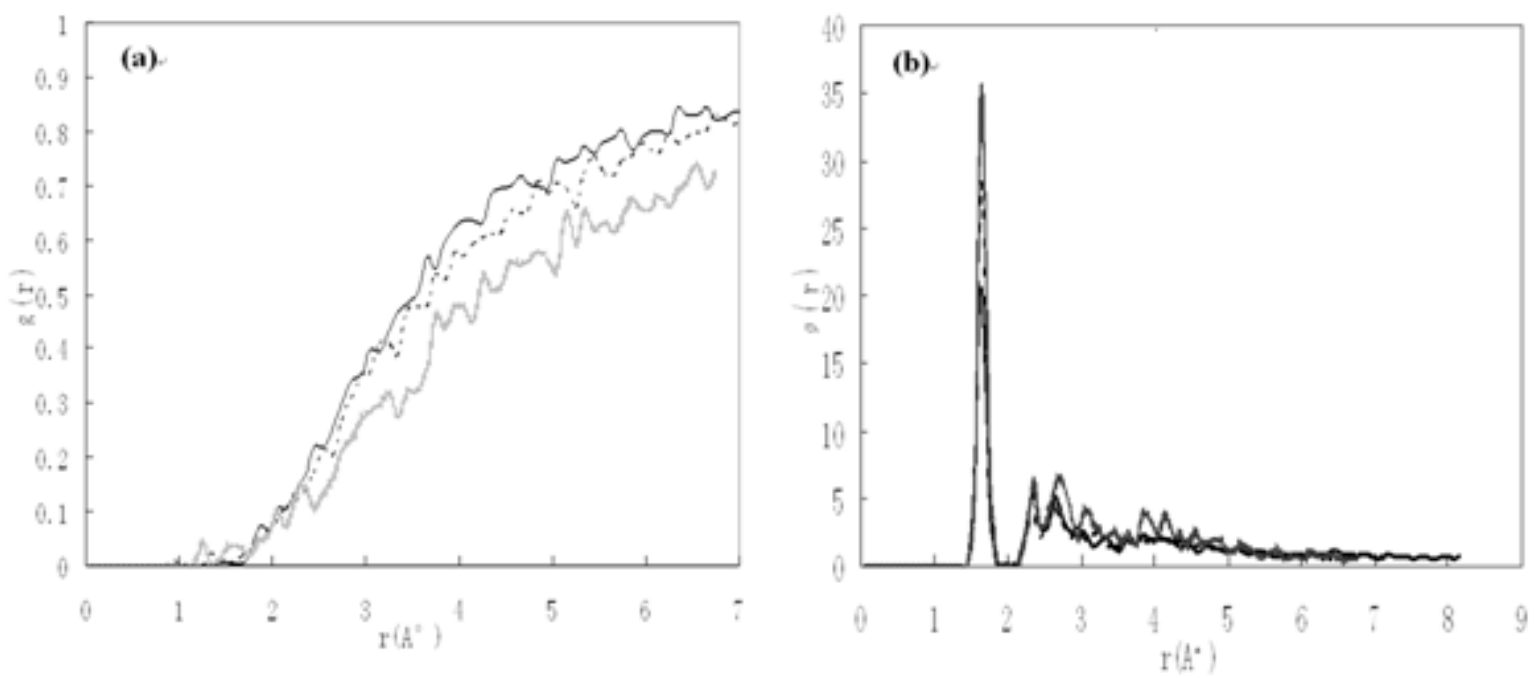

FIGURE III. PAIR CORRELATION FUNCTIONS G(R) FO 6PU (SOLID LINE), 12PU (DOTTED LINE) AND 20PU (DASHED LINE): (A) BASED ON ATOMS (SI, O ) OF THE TSP-POSS CORES TO THE ATOMS IN THE MAIN CHAINS OF HYBRID COMPOSITES; (B) BASED ON ALL BACKBONE ATOMS OF HYBRID COMPOSITES.

C. Mobility Of Fractures In Polymer Chains In Tsp-Poss/Pu Hybrid Composites

The mobility of the atoms (molecules) in a simulation system is investigated by using mean square displacement (MSD), which can be computed from Eq.(1). [11]

$$
\operatorname{MSD}=\left[\mid\left(r_{i}(t)-r_{i}(0) \mid{ }^{2}\right] .\right.
$$

Where $r(0)$ is the initial positional coordinate of atom $i$ (or molecules) and ri(t) is the coordinates of time.

Thus, the motion restrictions of polymer chains imposed by TSP-POSS structure can be evaluated by elucidating the mobility of atoms in polymer chains with and without the
TSP-POSS incorporation. In Figure4a-c, the MSD of all atoms in the TSP-POSS/PU samples is compared to that of the Si-O pairs in TSP-POSS cores. Whatever concentration is, the MSD of TSP-POSS core is all lower than that of all atoms as a whole. This result confirms that the TSP-POSS cage linked to polymer backbones acts as a heavy core with less mobility. In Figure 4d, it shows the comparison of enclosed cage cores mobility with different TSP-POSS concentration in hybrid composites. From the plot, the behavior of TSP-POSS is non-diffusive and stationary as MSD lower than $5 \mathrm{~A}^{2}$. Meanwhile, as the concentration of TSP-POSS increasing, the value of MSD decreases due to the motion restriction from humping cage reunion. 

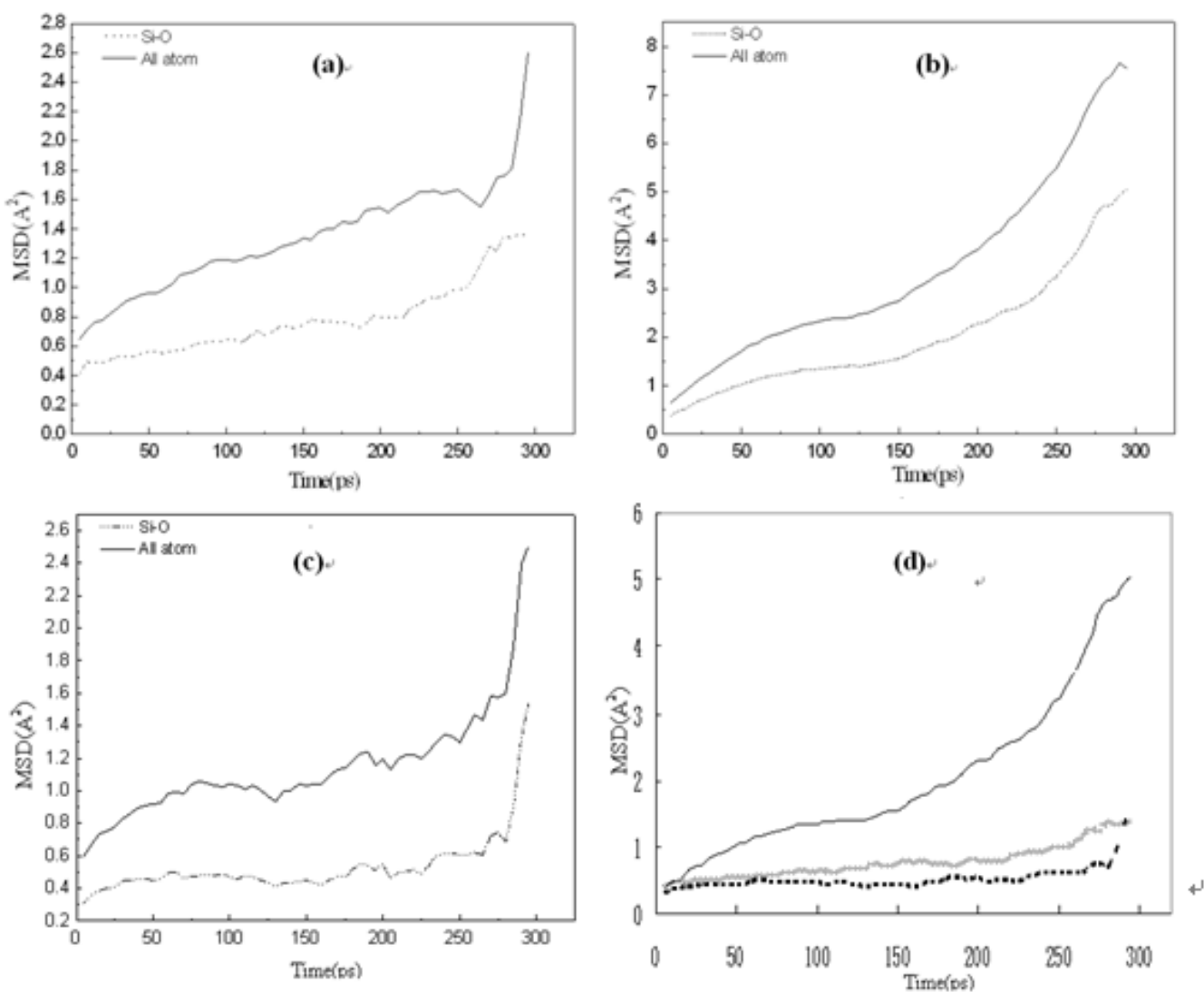

FIGURE IV. MEAN SQUARE DISPLACEMENT OF ALL ATOMS IN COMPOSITES (SOLID LINES) AND SI-O PAIRS IN TSP-POSS CORES (DOTTED LINES): (A).6PU; (B).12PU; (C).20PU. (D) MEAN SQUARE DISPLACEMENT OF SI-O PAIRS OF TSP-POSS CORES IN 6PU (DOTTED LINE), 12PU (SOLID LINE) AND 20PU (DASHED LINE).

\section{SUMMARY}

In this study, molecular simulation was applied in study the effect of TSP-POSS cage structure on domain micro-structure of TSP-POSS/PU hybrid composites at molecular level. The fluctuation of density and optimized energy plots verified the accuracy of the models and the applied force field. As the result shows, with TSP-POSS concentration increasing in hybrid composites, polymer chains contacts is decreased due to the presence of humping cage structure in TSP-POSS fractures while cage cores are inclined to be closer to each other. Meanwhile, a significant decrease in the mobility of TSP-POSS cores and polymer backbones can be observed, which demonstrates that TSP-POSS cage, as a rigid core linked to the TSP-POSS/PU backbone, apparently restricts the motion of the whole polymer chain.

\section{REFERENCES}

[1] Information on http://www.hybridplastics.com/docs/user-v2.06.pdf

[2] Information on http://en.wikipedia.org/wiki/Polyurethane

[3] Jianqing Zhao, Yi Fu and Shumei Liu, Polyhedral oligomeric silsesquioxane (POSS)-Modified thermoplastic and thermosetting nanocomposites: A review.,Polym. Polym. Compos. 16(8)(2008) 483-500.

[4] Zheng L., Farris R.J. and Coughlin E.B., Novel Polyolefin Nanocomposites:Synthesis and Characterizations of Metallocene-Catalyzed Polyolefin Polyhedral Oligomeric Silsesquioxane Copolymers, Macromol.34(2001) 8034-8039.
[5] Waddon A.J., Zheng L., Farris R.J. and Coughlin E.B., Nanostructured Polyethylene-POSS Copolymers:Control of Crystallization and Aggregation., Nano Lett. 2(10)(2002) 1149-1155

[6] Zheng L., Waddon A.J., Farris R.J. and Coughlin E.B., X-ray Characterizations of Polyethylene Polyhedral Oligomeric Silsesquioxane Copolymers. ,Macromol.35(2001)2375-2379.

[7] Samy A. Madbouly, Joshua U. Otaigbe, Recent advances in synthesis, characterization and rheological properties of polyurethanes and POSS/polyurethane nanocomposites dispersions and films., Prog. Polym. Sci. 34(2009)1283-1332.

[8] Lingling Wang, Ming Zhang, Yong Liu and Rui Pan, Influence of trisilanolphenyl POSS on structure and thermal properties of polyurethane hybrid composites: a molecular simulation approach, Acta Polymerica Sinica. Doi: 10.11777/j.issn1000-3304.2015.14231

[9] Matthew Oaten and Namita Roy Choudhury, Silsesquioxane-urethane hybrid for thin film applications., Macromol. 38(2005) 6392-6401

[10] Stéphane Bizet, Jocelyne Galy Molecular dynamics simulation of organic-inorganic copolymers based on methacryl-POSS and methyl methacrylate., Polym. 47(2006) 8219-8227.

[11] Bruce X Fu, Benjamin S Hsiao, Joseph Schwab et al., Nanoscale reinforcement of polyhedral oligomeric silsesquioxane (POSS) in polyurethane elastomer., Polym. Int. 49(2000) 437-440 